\title{
AVALIAÇÃO DOS RESULTADOS CLÍNICOS E RADIOGRÁFICOS DE PACIENTES SUBMETIDOS À RESSECÇÃO DE HEMIVÉRTEBRA NAS DEFORMIDADES CONGÊNITAS DA COLUNA VERTEBRAL
}

\author{
EVALUATION OF CLINICAL AND RADIOGRAPHIC RESULTS IN PATIENTS UNDERGOING \\ RESECTION OF HEMIVERTEBRA IN CONGENITAL DEFORMITIES OF THE SPINE \\ EVALUACIÓN DE LOS RESULTADOS CLÍNICOS Y RADIOGRÁFICOS DE LOS PACIENTES \\ SOMETIDOS A RESECCIÓN DE HEMIVÉRTEBRA EN DEFORMIDADES CONGÉNITAS \\ DE LA COLUMNA VERTEBRAL
}

Luis Antonio Medeiros Moliterno', Alderico Girão de Barros², América Maria limoeiro³, André luiz loyelo Barcellos³, Antonio Eulálio Pedrosa de Araújo Junior ${ }^{3}$, Renato Henriques Tavares ${ }^{3}$, Luiz Claudio Schettino ${ }^{4}$, Luis Eduardo Carelli Teixeira da Silva ${ }^{5}$

\begin{abstract}
RESUMO
Objetivo: A proposta do estudo foi determinar nossos resultados clínicos e radiográficos de pacientes com deformidades congênitas da coluna vertebral submetidos à ressecção de hemivértebra por via posterior isolada e correção com instrumentação posterior e fusão. Métodos: Registros de 31 pacientes submetidos à ressecção de hemivértebra no período de 2003 a 2010 foram revistos para identificar idade e sexo, grau de correção, níveis da artrodese, quadro neurológico, perda sanguínea, tempo cirúrgico e complicações. Resultados: Foram identificados 22 pacientes com escoliose e Cobb pré-operatório médio de $46.66^{\circ}\left(20-88^{\circ}\right)$ e 9 pacientes com cifose e média angular de $83.54^{\circ}\left(13-137^{\circ}\right)$. Vinte e quatro pacientes eram do sexo feminino e 7 do sexo masculino. Foram 13 pacientes adolescentes e 18 crianças (1-19 anos). A ressecção de hemivértebra foi realizada em 1 nível (64\%), 2 níveis (32\%) e 3 níveis (4\%). As taxas de correção da escoliose e cifose foram 63.8 e $40.1 \%$ e as médias angulares pós-operatórias foram $16.88^{\circ}$ e $50^{\circ}$, respectivamente. Complicações ocorreram em 7 pacientes: pseudartrose, cifose juncional, neurite óptica, déficit neurológico, infecção de ferida operatória e óbito. A média de perda sanguínea foi de $1132 \mathrm{ml}(300 \mathrm{ml}-3500 \mathrm{ml})$ e o tempo cirúrgico foi de 7.15 horas (4-13 horas). Conclusões: A ressecção de hemivértebra é uma valiosa técnica no tratamento das deformidades congênitas angulares e uma alternativa eficiente que oferece correção satisfatória nos planos coronal e sagital sem a necessidade de uma abordagem anterior.
\end{abstract}

Descritores: Coluna vertebral/anormalidades; Coluna vertebral/cirurgia; Escoliose/congênito; Procedimentos ortopédicos/métodos.

\begin{abstract}
Objective: The purpose of this study was to determine our clinical and radiographic results of patients with congenital deformities of the spine underwent to single-stage posterior hemivertebra resection and correction with segmental posterior instrumentation and fusion. Methods: The records of 31 consecutive patients who had undergone to hemivertebra resection between 2003 and 2010 were reviewed to identify age and sex, correction rates, fusion levels, neurological status, blood loss, time of surgery and complications. Results: We identified 22 patients with scoliosis and pre-operative Cobb averaged $46.66^{\circ}$ (range 20-88 ) and 9 patients with kyphosis and averaged $83.54^{\circ}$ (range $\left.13-137^{\circ}\right)$. Twenty four patients were female and 7 were male. Thirteen patients were teenagers and 18 were children (range $1-19$ years old). The hemivertebrae resection was done in 1 level (64\%), 2 levels (32\%) and 3 levels (4\%). The correction rates for scoliosis and kyphosis were $63.8 \%$ and $40.1 \%$ and pos-operative Cobb averaged was $16.88^{\circ}$ and $50^{\circ}$. Complications occurred in 7 patients: pseudoarthrosis, junctional kyphosis, optic neuritis, neurologic deficit, wound infection, and death. The average blood loss was $1132 \mathrm{ml}$ (range $300 \mathrm{ml}-3500 \mathrm{ml}$ ) and time of the surgery was 7.15 hours (range 4-13 hours). Conclusions: Posterior hemivertebra resection is a valuable technique to assessment angular congenital spinal deformities and an efficient alternative that offers satisfactory correction in both coronal and sagittal planes without need for anterior approach.
\end{abstract}

Keywords: Spine/abnormalities; Spine/surgery; Scoliosis/congenital; Orthopedic procedures/methods.

\section{RESUMEN}

Objetivo: El objetivo de nuestro estudio fue determinar los resultados clínicos y radiográficos de los pacientes con deformidades congénitas de la columna vertebral que se sometieron a la resección de hemivértebra por el acceso aislado posterior con instrumentación posterior y fusión. Métodos: Registros de 31 pacientes sometidos a resección de la hemivértebra el período 2003 a 2010 fueron revisados para identificar edad y sexo, grado de corrección, niveles de la fusión, estado neurológico, pérdida de sangre, tiempo quirúrgico y complicaciones. Resultados: Se identificaron 22 pacientes con escoliosis y Cobb promedio preoperatorio de $46,66^{\circ}\left(20^{\circ}\right.$ - $\left.88^{\circ}\right)$ y 9 pacientes con un ángulo medio de cifosis y $83,54^{\circ}\left(13^{\circ}-137^{\circ}\right)$. Veinticuatro pacientes eran hombres y siete mujeres. Hubo 13 adolescentes y 18 niños (1-19 años).

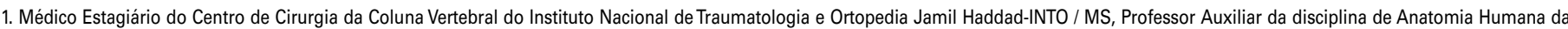
Universidade Estácio de Sá - Rio de Janeiro, RJ, Brasil.

2. Médico Estagiário do Centro de Cirurgia da Coluna Vertebral do Instituto Nacional de Traumatologia e Ortopedia Jamil Haddad-INTO / MS - Rio de Janeiro, RJ, Brasil.

3. Médico Ortopedista do Centro de Cirurgia da Coluna Vertebral do Instituto Nacional de Traumatologia e Ortopedia Jamil Haddad-INTO / MS - Rio de Janeiro, RJ, Brasil.

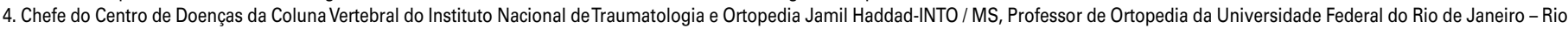
de Janeiro, RJ, Brasil.

5. Chefe do Centro de Trauma Raquimedular do Instituto Nacional de Traumatologia e Ortopedia Jamil Haddad-INTO / MS - Rio de Janeiro, RJ, Brasil.
}

Trabalho realizado no Instituto Nacional deTraumatologia e Ortopedia Jamil Haddad-INTO - Rio de Janeiro, RJ, Brasil.

Correspondência: Rua:Clóvis Beviláqua 95/101.Tijuca. Rio de Janeiro. RJ. Brasil. CEP 20520-160. luismoliterno@yahoo.com.br 
La resección de hemivértebra se realizó en un nivel (64\%), dos niveles (32\%) y tres niveles (4\%). Los porcentajes de corrección de la escoliosis y la cifosis fueron 63,8\% y el 40,1\% y el ángulo de post-operatorio promedio fue de $16.88^{\circ}$ y $50^{\circ}$, respectivamente. Se presentaron complicaciones en 7 pacientes, pseudoartrosis, cifosis de la unión, neuritis óptica, déficit neurológico, infección de la herida y muerte. La pérdida de sangre promedio fue de $1132 \mathrm{ml}$ (300 ml-3500 ml) y el tiempo quirúrgico medio fue de 7,15 horas (4-13 horas). Conclusiones: La resección de hemivértebra es una técnica valiosa en el tratamiento de deformidades angulares congénitas y una alternativa eficaz que proporciona corrección satisfactoria en el plano coronal y sagital sin la necesidad de una vía anterior.

\section{Descriptores: Columna vertebral/anomalías; Columna vertebral/cirugía; Escoliosis/congénito; Procedimientos ortopédicos/métodos.}

\section{INTRODUÇÃO}

A etiologia congênita representa $5 \%$ a $12 \%$ das escolioses, três quartos são progressivas e evoluem para deformidades inaceitáveis, se não tratadas corretamente ${ }^{1}$. As deformidades congênitas da coluna vertebral ocorrem no embrião, em torno da $5^{\mathrm{a}}$ ou da $6^{\mathrm{a}}$ semana, época crítica no desenvolvimento da coluna, quando as estruturas anatômicas da coluna se formam no mesênquima e ocorrem os processos de segmentação ${ }^{2,3}$. Podem ocorrer dois tipos básicos de distúrbios nessa fase: falhas de formação de uma ou mais vértebras e falhas de segmentação ${ }^{4}$.

Escoliose congênita é uma curvatura lateral da coluna causada pela presença de anomalias vertebrais que resultam em um desequilíbrio do crescimento longitudinal. Uma vez que a escoliose congênita muitas vezes é rígida e a correção pode ser difícil, é importante detectar essas curvas precocemente e instituir tratamento apropriado enquanto a curva é pequena, em vez de tentar procedimentos mais complexos que são necessários quando a deformidade é grave ${ }^{3,5}$. Acredita-se que a maioria das escolioses congênitas são causadas por fatores ambientais fetais não genéticos, porém, não detectáveis pelo histórico.

Foram descritas opções de tratamento cirúrgico como fusões posteriores com ou sem instrumentação, fusões anteriores e posteriores combinadas, hemiepifisiodese convexa anterior, instrumentação sem fusão e mais recentemente a ressecção de hemivértebra. Inicialmente descrita por Leatherman e Dickson ${ }^{6}$ e popularizada por Bradford ${ }^{7,8}$ no final da década de 80 , a ressecção vertebral constitui opção nas deformidades rígidas e graves. Bradford et al. ${ }^{9}$ realizaram a técnica por abordagem circunferencial obtendo excelente correção da deformidade e balanço do tronco. Ruf e Harms et al. ${ }^{10}$, Suk et al. ${ }^{11,12}$ descreveram a vertebrectomia (VCR) por via posterior isolada em pacientes com escoliose rígida.

\section{OBJETIVOS}

O presente estudo tem como objetivo determinar os resultados clínicos e radiográficos de pacientes com diagnóstico de deformidades congênitas da coluna vertebral submetidos à ressecção de hemivértebra por via posterior isolada.

\section{MATERIAIS E MÉTODOS}

Uma análise retrospectiva dos prontuários e de exames de imagem pré e pós-operatórios e avaliação clínica de pacientes submetidos à ressecção de hemivértebra por via posterior isolada no período de 2003 a 2010 foi realizada para identificar os resultados clínicos e radiográficos da osteotomia de ressecção vertebral por via posterior isolada em deformidades congênitas da coluna. As informações dos pacientes selecionados previamente foram obtidas no Setor de Arquivos Médicos do Instituto Nacional de Traumatologia e Ortopedia Jamil Haddad-INTO/MS após aprovação no Comitê de Ética em Pesquisa da nossa instituição. Os casos foram avaliados através da análise, inicialmente, de 36 prontuários sendo incluídos os pacientes submetidos à ressecção vertebral por acesso posterior e instrumentação pedicular posterior e fusão com diagnóstico de escoliose e/ou cifose de etiologia congênita, pelos médicos cirurgiões do Centro de Doenças da Coluna Vertebral do Instituto Nacional de Ortopedia e Traumatologia Jamil Haddad - INTO/MS.

Foram excluídos, previamente, aqueles com etiologias idiopática, neuromuscular, pós-traumática e tumoral e aqueles que, durante a coleta das informações, não se observou adequado acompanhamento no período pós-operatório proposto, validando um total de 31 casos para o estudo. As cirurgias foram realizadas pelos médicos ortopedistas do Centro de Doenças da Coluna Vertebral do Instituto Nacional de Traumatologia e Ortopedia Jamil Haddad-INTO/MS utilizando a mesma técnica operatória.

As variáveis estudadas foram idade à época da cirurgia, sexo, ângulos de Cobb pré e pós-operatórios, quadro neurológico de acordo com a escala de Frankel, a deformidade, o tempo cirúrgico, a perda sanguínea per-operatória, complicações, nível(s) do defeito congênito, níveis instrumentados e submetidos à fusão.

Foram determinados os valores angulares das curvas principais e secundárias nos planos coronal e sagital pelo método de Cobb antes e após os procedimentos cirúrgicos através de radiografias panorâmicas nas incidências ântero-posterior e perfil. O tempo de cirurgia e a perda sangüínea per-operatória foram obtidas com base nas fichas anestésicas. A identificação das complicações ocorridas durante o seguimento dos casos foram obtidas a partir dos registros dos prontuários, assim como, a determinação do quadro neurológico realizada na avaliação pré-operatória e durante o acompanhamento ambulatorial no período pós-operatório.

\section{TÉCNICA CIRÚRGICA}

O paciente é posicionado em decúbito ventral na mesa cirúrgica.Inicia-se acesso longitudinal posterior procedendo-se, a seguir, à instrumentação pedicular segmentar cranial e caudal ao nível da osteotomia. Terminada a instrumentação, os corpos vertebrais a serem ressecados são acessados através de costotransversectomia quando na região torácica ou abordagem posterior circunferencial na coluna lombar. O descolamento subperióstico é preferível e quando necessária é feita a ligadura dos vasos segmentares e/ou raízes nervosas no nível dorsal. Optamos pela utilização de cola de fibrina para hemostasia dos vasos epidurais expostos após a laminectomia. Estabiliza-se um dos lados da deformidade com barra antes de se proceder à osteotomia para que não ocorra movimento translacional. Em casos de deformidades cifóticas ou quando o espaço entre os corpos vertebrais remanescentes for maior que $5 \mathrm{~mm}$, a utilização de espaçador de titânio (cesta) para o suporte interssomático anterior preenchido com enxerto autólogo das costelas ressecadas e dos corpos vertebrais se faz necessário. Durante as manobras de correção a monitorização com potencial evocado motor (PEM) informa alterações da condução nervosa. Quando não dispusemos da monitorização foi realizado o teste de despertar. Indicamos a utilização de colete por três meses a todos os pacientes.

\section{RESULTADOS}

Entre os pacientes selecionados, 13 eram adolescentes (idade superior a 12 anos) e 18 crianças. Em relação ao sexo, 24 eram meninas e sete eram meninos, em uma proporção de 3,4:1. Quanto às deformidades, foram 22 escolioses, predominantemente, com média angular pré-operatória da curva principal de $46.66^{\circ}\left(20-88^{\circ}\right)$ e $26.54^{\circ}\left(10-58^{\circ}\right)$ das curvas secundárias e nove cifoses com média angular pré-operatória da curva principal de $83.54^{\circ}\left(13^{\circ}-137^{\circ}\right)$ e $35.5^{\circ}\left(20-51^{\circ}\right)$ das curvas secundárias. As medidas dos ângulos de Cobb pós-operatórios das curvas escolióticas e cifóticas principais foram, em média, $16,88^{\circ}$ e $50^{\circ}$ respectivamente, o que corresponde a uma taxa de correção de 63,8\% e $40.1 \%$. A taxa de correção das curvas secundárias nas escolioses foi de $68.72 \%\left(9-18^{\circ}\right)$ e nas cifoses, $75 \%\left(5-15^{\circ}\right)$. 
O tempo de cirurgia variou de 4 a 13 horas, com uma média de 7.15 horas. O maior tempo cirúrgico ocorreu em um paciente do sexo feminino de 19 anos de idade com escoliose e instrumentação de T1 a L3 enquanto o menor procedimento ocorreu em um paciente do sexo feminino e 17 anos de idade com escoliose congênita instrumentada de T5 a T8. A perda sanguínea variou de $300 \mathrm{ml}$ em um tempo cirúrgico de 4 horas à $3500 \mathrm{ml}$ em uma escoliose que durou 13 horas e que apresentou infecção da ferida cirúrgica no pós-operatório. A média de perda sanguínea per-operatória foi de $1132 \mathrm{ml}$.

As ressecções de hemivértebras foram realizadas em apenas um segmento em 20 (64\%) pacientes, em dois segmentos em 10 (32\%) e em três segmentos em um (4\%) paciente sendo 22 ressecções na coluna torácica e nove na coluna lombar. Foi necessária utilização de cestas intersomáticas em 12 casos.

Complicações ocorreram em sete pacientes. Cifose juncional com repercussão clínica foi observada em um paciente do sexo feminino de 12 anos de idade com diagnóstico de escoliose cujo nível do defeito congênito era T3. Infecção da ferida operatória e necessidade de desbridamento cirúrgico ocorreram em dois pacientes. Pseudartrose foi diagnosticada em um paciente de cinco anos de idade com escoliose congênita e Cobb pré-operatório de $70^{\circ}$. O mesmo paciente apresentou ainda descência de ferida no pós-operatório imediato. Observamos um caso de neurite óptica unilateral em uma paciente de 19 anos e escoliose congênita após procedimento de ressecção dos níveis T5-T6. A maior perda sanguínea desta série ocorreu neste caso. Até o momento a paciente permanece com perda da acuidade visual unilateral.
Tivemos um caso de óbito no período pós-operatório tardio não relacionado ao procedimento cirúrgico devido à pneumonia comunitária.

O quadro neurológico pré-operatório de 28 pacientes foi classificado como Frankel E e mantiveram-se nesta escala durante o período pós-operatório. Um paciente do sexo masculino, 14 anos apresentava-se à avaliação neurológica inicial como Frankel B, evoluindo em três meses de acompanhamento ambulatorial para Frankel E. Em um paciente com cifose congênita de $97^{\circ}$ houve progressão de Frankel C para E. Entretanto, tivemos um caso de paresia bilateral (L4,L5 e S1) presente no pré-operatório e que permaneceu inalterado após o procedimento cirúrgico em um paciente com cifose congênita de $109^{\circ}$

Os resultados obtidos com a ressecção de hemivértebra por via posterior isolada encontram-se na Tabela 1.

\section{DISCUSSÃO}

Malformações congênitas são responsáveis por deformidades na coluna vertebral e seu tratamento cirúrgico é desafiador. Os desequilíbrios nos planos coronal, sagital e axial proporcionam a progressão da deformidade, comprometem a função cárdiopulmonar, e eventualmente, acarretam déficits neurológicos ${ }^{1,2}$. Técnicas cirúrgicas têm sido objeto de estudo por inúmeros autores $^{9-16}$, incluindo-se as ressecções de hemivértebras, as osteotomias e as reconstruções anterior e posterior para correção das deformidades.

A primeira ressecção de hemivértebra foi realizada por Royle

Tabela 1. Resultados dos pacientes submetidos à ressecção de hemivértebra por via posterior isolada. *

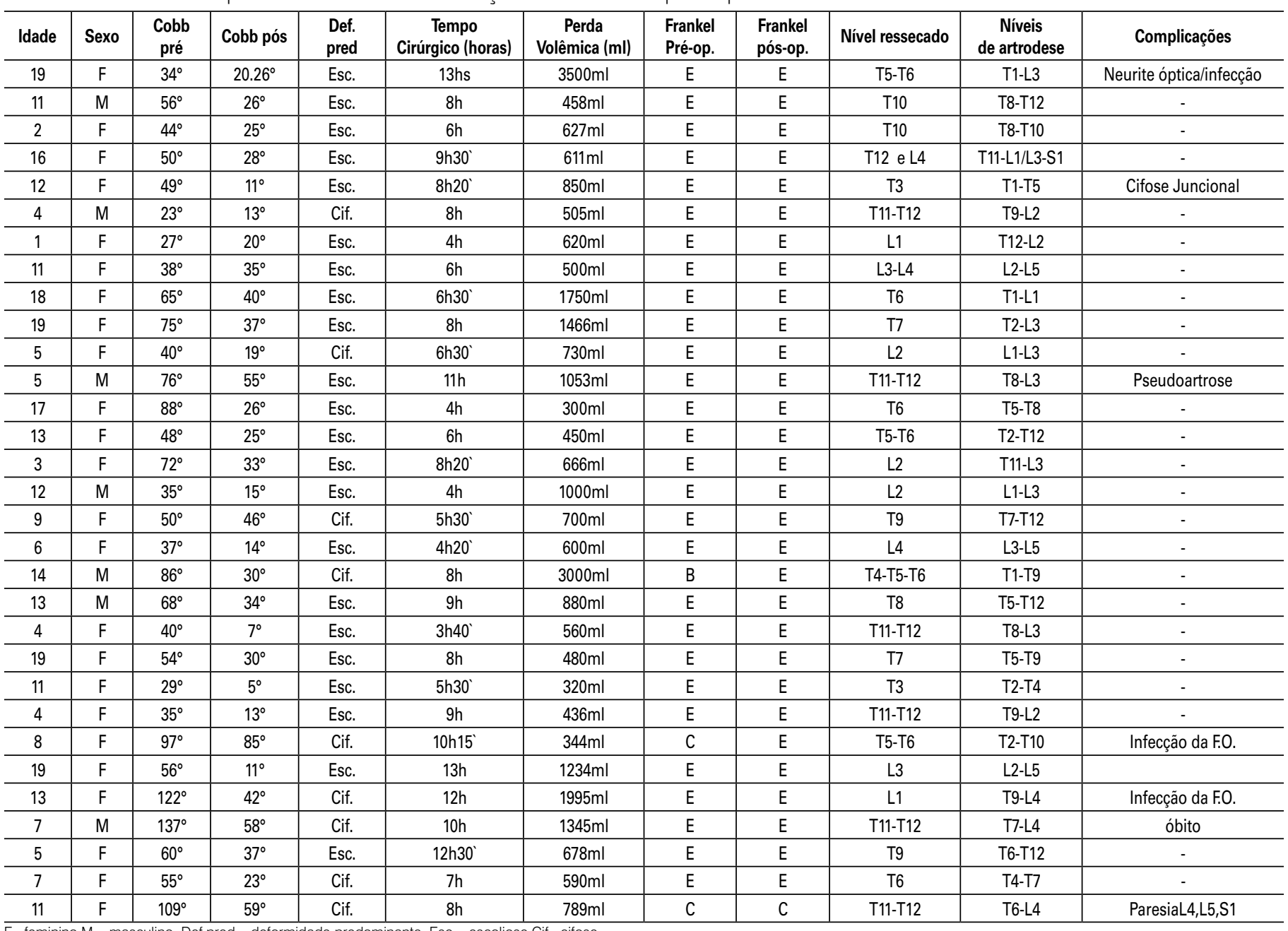

$\mathrm{F}=$ feminino, $\mathrm{M}=$ masculino, Def. .pred. $=$ deformidade predominante, $\mathrm{Esc}=$ escoliose,Cif=cifose.

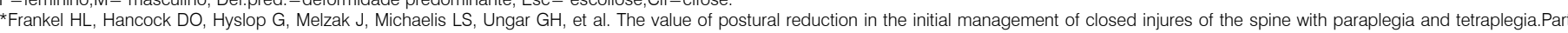
1.Paraplegia 1969;7:179-92. 
em $1921^{17}$. MacLennam ${ }^{18}$ descreveu em 1922 a ressecção apical associada à imobilização gessada pós-operatória em escolioses rígidas. A abordagem circunferencial com liberação das estruturas anteriores e fusão seguida de instrumentação e fusão posteriores foi primeiramente descrita por Bradford ${ }^{8}$. Em seu estudo em 13 pacientes, obteve $52 \%$ de correção. Entretanto, relatou tempo cirúrgico médio de 12 horas e perda volêmica média de 5500ml.

A utilização da técnica de ressecção de hemivértebra pela via posterior isolada, no tratamento das deformidades congênitas proporciona boa correção e estabilização, embora tecnicamente exigente. De acordo com Suk, é indicada nas deformidades com valor angular maior que $80^{\circ}$ e flexibilidade menor que $25 \%$ nos testes de inclinação lateral ${ }^{11-16}$. Em seu estudo de 16 pacientes com ressecção de hemivértebra por acesso posterior foi obtida correção de 59\%, porém, um caso evoluiu com paralisia completa. Lenke et al. ${ }^{19}$ utilizando a via posterior isolada para ressecção de hemivértebra em 35 crianças obteve taxa de correção de $51 \%$ em escoliose grave, $55 \%$ nos casos de cifose global, $58 \%$ em cifose angular e $54 \%$ nas cifoescolioses.

Em nossa casuística obtivemos taxa de correção média de 52\% utilizando a mesma abordagem em concordância com a literatura que relata taxas de $51 \%$ a $59 \%{ }^{11,19}$.

A ressecção de hemivértebra por acesso combinado permite maior controle das colunas anterior e posterior, porém, requer tempo cirúrgico maior e está associado à perda sanguínea mais expressiva, além de maior impacto na função respiratória ${ }^{11}$. Entretanto, a via posterior isolada constitui um procedimento em estágio único o que implica em menores tempo cirúrgico e perda volêmica, maior estabilização e reconstrução através do uso de suporte na coluna anterior, resulta em melhor função pulmonar pós-operatória e permite correção e balanço do tronco de forma mais efetiva ${ }^{11,12,20}$. De acordo com Jalanko et al. ${ }^{20}$ pacientes submetidos à abordagem posterior isolada tendem a apresentar melhor escore no questionário de qualidade de vida (Scoliosis Research Society [SRS]-24) imediatamente após a cirurgia, porém, não há diferença significante durante o longo seguimento ${ }^{21}$. A abordagem combinada muitas vezes requer uma toracotomia o que implica em drenagem pleural após o procedimento cirúrgico, quadro álgico mais expressivo e resultado estético menos satisfatório.

Anatomicamente irrigação medular é menor entre os níveis de T4 a T9 e a ligadura das artérias segmentares ao nível da ressecção da hemivértebra pode ocasionar isquemia do tecido nervoso. De acordo com alguns autores, a interrupção do suprimento sanguíneo em até quatro níveis não traz prejuízo à função neurológica ${ }^{22-24}$ Em nosso estudo ligaduras arteriais foram realizadas em até três níveis sem repercussão neurológica $O$ encurtamento medular após a ressecção vertebral também apresenta risco ao desenvolvimento de lesão neurológica por alteração de sua morfologia e redução da irrigação sanguínea. Ueda et al. ${ }^{24}$ relataram, em experimentos em cães, que o encurtamento maior que $12.5 \mathrm{~mm}$ acarreta compressão da medula na concavidade pelo saco dural redundante além de proporcionar o "acotovelamento" medular e quando superiores a $20 \mathrm{~mm}$ acarretam anormalidades ao PEM. Em nossa prática optamos pela utilização das cestas anteriores em 12 casos, quando o espaço remanescente entre os corpos vertebrais foi superior a $5 \mathrm{~mm}$, com objetivo de prevenir encurtamento excessivo e para auxiliar na correção de cifose ${ }^{11}$. Durante as manobras de correção há grande risco de lesão neurológica. A utilização de PEM e manutenção da PAM em $75-80 \mathrm{mmHg}$ são importantes neste momento do ato cirúrgico. Perda de $19 \%$ de sinal intra-operatório foi relatada na literatura ${ }^{25}$. Nenhum paciente em nossa casuística evoluiu com déficit neurológico no per-operatório.

Neurite óptica isquêmica é uma rara complicação pós-operatória da cirurgia da coluna vertebral a qual pode estar associada à posição prona por período superior a 5 horas e perda sanguínea superior a 1 litro ${ }^{26}$. Embora ocorra mais comumente em homens entre 30 e 69 anos, em nossa série foi observado um caso que ocorreu em uma paciente de 19 anos com escoliose congênita. A instrumentação e fusão foi realizada de T3 a L1. O tempo cirúrgico neste caso foi de 13 horas e a perda sanguínea de $3500 \mathrm{ml}$. A literatura acerca desta complicação ainda apresenta baixo índice de evidencia e é, sobretudo, composta de relato de casos. Devido à complexidade das ressecções vertebrais quanto ao tempo cirúrgico e à perda sanguínea, torna-se uma potencial complicação nesta técnica operatória.

\section{CONCLUSÃO}

A ressecção de hemivértebra por via posterior isolada é um procedimento complexo e depende de uma equipe experiente. Atualmente é considerada uma opção efetiva nas deformidades congênitas encontrando sua melhor indicação nas deformidades estruturadas da coluna vertebral.

\section{REFERÊNCIAS}

1. Winter RB. Congenital scoliosis. Orthop Clin North Am. 1988; 19(2):395-408.

2. McMaster MJ, Ohtsuka K. The natural History of congenital scoliosis. A study of two hundred and fifty-one patients.J Bone Joint Surg Am. 1982:64(8):1128-47.

3. Winter RB, Moe JH, Eilers VE. Congenital scoliosis. A study of 234 patients treated and untreated. Part I. Natural history. J Bone Joint Surg Am. 1968;50(1):1-15.

4. Nasca RJ, Stilling FH, Stell HH. Progression of congenital scoliosis due to hemivertebrae and hemivertebrae with bars. J Bone Joint Surg Am. 1975;57(4):456-66.

5. Winter RB, Moe JH, Lonstein JE. Posterior spinal arthrodesis for congenital scoliosis. An analysis of the cases of two hundred and ninetypatients, five to nineteen years old. $J$ Bone Joint Surg Am. 1984;66(8):1188-97.

6. Leatherman KD, Dickson RA. Two stage corrective surgery for congenital deformities of the spine. J Bone Joint Surg Br. 1979:61(3):324-8.

7. Bradford DS, Tribus CB. Vertebral column resection for the treatment of rigid coronal decompensation. Spine (Phila Pa 1976). 1997;22(14):1590-9

8. Bradford DS. Vertebral column resection. Printed abstract from the association of Bone and Joint Surgeons Annual Meeting. Orthop Trans. 1987;11:502.

9. Bradford DS, Boachie-Adjei O. One stage anterior and posterior hemivertebral resection and arthrodesis for congenital scoliosis. J Bone Joint Surg Am. 1990;72(4):536-40.

10. Ruf M, Harms J. Hemivertebra resection by posterior approach-innovative operative technique and first results. Spine (Phila Pa 1976). 2002;27(10):1116-23.

11. Suk SI, Kim JH, Kim WJ, Lee SM,Chung ER, Nah KH. Posterior vertebral colunm resection for severe spinal deformities. Spine (Phila Pa 1976). 2002;27(21):2374-82.

12. Suk SI, Chung ER, Kim WJ, Kim SS, Lee JS, Choi WK. Posterior vertebral column resection for severe rigid scoliosis. Spine (Phila Pa 1976). 2005; 30(14):1682-7.

13. Ruf M, Harms J. Posterior hemivertebra resection with transpedicular Instrumentation: early correction in children aged 1 to 6 years. Spine (Phila Pa 1976). 2003; 28(18):2132-8.

14. Shono Y, Abumi K, Kaneda K. One stage posterior hemivertebra resection and correction using segmental posterior instrumentation. Spine (Phila Pa 1976). 2001;26(7): 752-7.

15. Shimode M, Kojima T, Sowa K. Spinal wedge osteotomy by a single posterior approach

for correction of severe and rigid kyphosis or kyphoscoliosis. Spine (Phila Pa 1976). 2002;27(20):2260-7.

16. Kawahara N, Tomita K, Baba H, Kobayashi T, Fujita T, Murakami H.Closing-opening wedge osteotomy to correct angular kyphotic deformity by a single posterior approach. Spine (Phila Pa 1976). 2001;26(4):391-402.

17. Royle ND. The operative removal of an accessory vertebra. Med J Aust. 1928;1:467-8.

18. MacLennan A. Scoliosis. BMJ. 1922;2:865-6.

19. Lenke LG, O'Leary PT, Bridwell KH, Sides BA, Koester LA, Blanke KM. Posterior vertebral column resection for severe pediatric deformity: minimum two-year follow-up of thirtyfive consecutive patients. Spine (Phila Pa 1976). 2009;34:2213-21.

20. Jalanko T, Rintala $R$, Puisto $V$, Helenius I. Hemivertebra resectionfor congenital scoliosis in young children. Spine (Phila Pa 1976). 2010;36(1):41-9.

21. Altiok H, Khanna N, Lubicky JP. Comparison of Anterior / Posterior and Posterior only approaches for Hemivertebrae excision in congenital scoliosis. In: SRS 39th Annual Meeting, Buenos Aires, Argentina, 2004

22. Kawahara N, Tomita K, kobayashi T, Abdel-Wanis ME, Murakami H, Akamaru T. influnce of acute shortening on the spinal cord: an experimental study. Spine (Phila Pa 1976). 2005:30(6):613-620.

23. Nambu K, Kawahara N, Kobayashi T,Murakami H, Ueda Y, Tomita K. Interruption of the bilateral segmental arteries at several levels: Influence on vertebral blood flow. Spine (Phila Pa 1976). 2004;29(14):1530-4.

24. Ueda Y, Kawahara N, Tomita K, Kobayashi T, Murakami H, Nambu K. Influence on spinal cord blood flow and function by interruption of bilateral segmental arteries up to three levels: experimental study in dogs. Spine (Phila Pa 1976). 2005;30(20):2239-43.

25. Cheh G, Lenke LG, Kim YJ, DaubsMD, Padberg AM, Kuhns CA, et al. Loss of spinal cord monitoring signals in children during thoracickyphosis correction with spinal osteotomy: Why does it occur and what should you do? [abstract]. EurSpine J. 2006; 15(Suppl 4):9467. [EuroSpine 2006. 8th Annual Meeting of the European SpineSociety, 25-28 October 2006 Istanbul, Turkey-Abstracts].

26. Lee LA, Newman NJ, Wagner TA, Dettori JR, Dettori NJ. Postoperative ischemic optic neuropathy. Spine (Phila Pa 1976). 2010;35(9S):S105-S16. 